\title{
Measurement of the differential cross sections of the processes with a direct photon and associated hadronic jet in $p \bar{p}$ collisions at $\sqrt{s}=1.96 \mathrm{TeV}$
}

\author{
Alexander Verkheev* (for the D0 collaboration) JINR \\ E-mail: alverejinr.ru
}

\begin{abstract}
The process $p \bar{p} \rightarrow \gamma+$ jet $+X$ is studied using $8.7 \mathrm{fb}^{-1}$ of integrated luminosity collected by the D0 detector at the Fermilab Tevatron Collider at a center-of-mass energy $\sqrt{s}=1.96 \mathrm{TeV}$. Photons are reconstructed with rapidities $\left|y^{\gamma}\right|<1.0$ or $1.5<\left|y^{\gamma}\right|<2.5$ and transverse momentum $p_{T}^{\gamma}>20 \mathrm{GeV}$. The highest- $p_{T}$ jet is required to be in one of the four rapidity regions up to $\left|y^{\text {jet }}\right| \leq 3.2$. The triple differential cross sections $\mathrm{d}^{3} \sigma / \mathrm{d} p_{T}^{\gamma} \mathrm{d} y^{\gamma} \mathrm{d} y^{\text {jet }}$ are measured as a function of $p_{T}^{\gamma}$ separately for events with the same sign $\left(y^{\gamma} y^{\text {jet }}>0\right)$ and opposite sign $\left(y^{\gamma} y^{\text {jet }} \leq 0\right)$ of photon and jet rapidities. Results are compared to next-to-leading order (NLO) perturbative QCD calculations using different sets of parton distribution functions and to predictions from SHERPA and PYTHIA Monte Carlo event generators. The NLO calculations are found to be in general agreement with the data, but do not describe all kinematic regions.
\end{abstract}

XXII International Baldin Seminar on High Energy Physics Problems

15-20 September, 2014

JINR, Dubna, Russia

\footnotetext{
* Speaker.
} 


\section{Introduction}

In hadron-hadron collisions the production of a direct photon with associated jets in the final state is a powerful probe of the dynamics of hard QCD interactions [1]. The term "direct" means that these photons do not result from mesons. Different angular configurations between the photon and the jets can be used to extend inclusive photon production measurements and provide information about the parton distribution functions (PDFs) of the incoming hadrons.

\section{Data selection}

The inclusive $\gamma+$ jet production cross section is measured differentially as a function of $p_{T}^{\gamma}$ in sixteen angular configurations of the leading jet and the photon rapidities for photons within rapidities $\left|y^{\gamma}\right|<1.0$ (central photons) or $1.5<\left|y^{\gamma}\right|<2.5$ (forward photons), while the jet is required to be in one of the four rapidity regions, $\left|y^{\text {jet }}\right| \leq 0.8,0.8<\left|y^{\text {jet }}\right| \leq 1.6,1.6<\left|y^{\text {jet }}\right| \leq 2.4$, or $2.4<\left|y^{\mathrm{jet}}\right| \leq 3.2$, and to satisfy the minimum transverse momentum requirement $p_{T}^{\mathrm{jet}}>15 \mathrm{GeV}$ [2]. These configurations are obtained by combining regions with positive and negative products of rapidities, $y^{\gamma} y^{\text {jet }}$. This selection allows one to probe PDFs in the range of parton momentum fractions $0.001 \leq x \leq 1$, and hard scatter scales of $400 \leq Q^{2} \equiv\left(p_{T}^{\gamma}\right)^{2} \leq 1.6 \times 10^{5} \mathrm{GeV}^{2}$. The measured cross sections are compared to the theoretical predictions from JETPHOX [3], PYTHIA [4], and SHERPA [5].

We have used a sample of $\gamma+$ jet events collected from June 2006 to September 2011 with the $\mathrm{D} 0$ detector in $p \bar{p}$ collisions at $\sqrt{s}=1.96 \mathrm{TeV}$ which correspond to an integrated luminosity of $8.7 \pm 0.5 f b^{1}$. The D0 detector is a general purpose detector described in detail elsewhere [6]. The events should pass triggers based on the identification of high pT cluster in the EM calorimeter with loose shower shape requirements for photons. Jets are reconstructed using the DO Run II iterative midpoint cone algorithm [7] with a cone size 0.7.

In total, approximately 7.2 (8.3) million $\gamma+$ jet candidate events with central (forward) photons are selected after application of all selection criteria. These samples include instrumental background contributions from electroweak interactions resulting in one or more electromagnetic clusters (from electrons or photons), and strong interactions producing a jet misidentified as a photon. The first type of background includes $W(\rightarrow e v)+$ jet, $Z / \gamma^{*}\left(\rightarrow e^{+} e^{-}\right)+$jet, and diphoton production. The combination of the track-match veto and $\#_{T}$ requirement (part of the photon identification criteria) reduces the contribution from the $W(\rightarrow e v)+$ jet process to a negligible level, less than $0.5 \%$ (1.5\%) for events with central (forward) photons. Contributions from $\mathrm{Z}+\mathrm{jet}$ and diphoton events are found to be even smaller. These backgrounds are subtracted from the selected data sample. To estimate the remaining background contribution from dijet events, a template fitting technique is employed [8] . The distributions of the artificial neural network (a group of variables exploiting the differences between the photon- and jet-initiated activity in the detector) output for the simulated photon signal and dijet background samples are fitted to the data for each $p_{T}^{\gamma}$ bin using a maximum likelihood fit to obtain the fractions of signal and background components in data. The $p_{T}^{\gamma}$ dependence of the signal fraction (purity) is fitted in each region using a three-parameter function, $P=a /\left(1+b\left(p_{T}^{\gamma}\right)^{c}\right)$. Fig. 1 shows the resulting purities for events with central photons, very central and very forward jet rapidities, for same-sign rapidities as an example. 

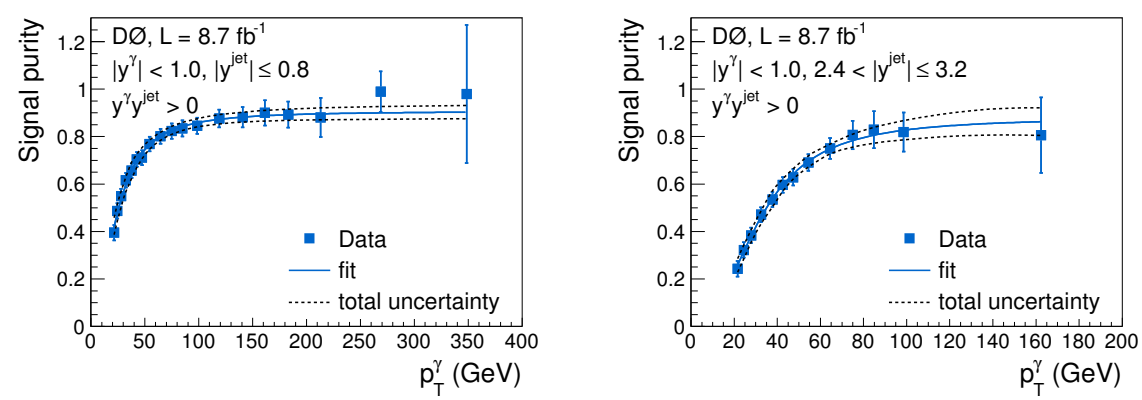

Figure 1: Purity of the selected $\gamma+$ jet sample as a function of $p_{T}^{\gamma}$, shown for central photons, very central and very forward jet rapidities, same-sign rapidity events. The solid line shows the fit and the dashed lines show the total fit uncertainty.

We calculate corrections to the observed rate of $\gamma+$ jet candidates to account for the photon and jet detection efficiencies (and for the geometric and kinematic acceptances) using samples of simulated $\gamma+$ jet MC events in which the photon is required to be isolated at the particle level by applying $p_{T}^{\text {iso }}<2.5 \mathrm{GeV}$. The acceptance typically varies within about $1.4-0.8$ with a relative systematic uncertainty of $3 \%-12 \%$, and takes into account the correlation between the samesign and opposite-sign events. The total efficiency of the photon selection criteria is $68 \%-80 \%$, depending on the $p_{T}^{\gamma}$ and $y^{\gamma}$ region with a relative systematic uncertainty of $3 \%(7.3 \%)$ for central (forward) photons.

\section{Results}

The differential cross section $\mathrm{d}^{3} \sigma / \mathrm{d} p_{T}^{\gamma} \mathrm{d} y^{\gamma} \mathrm{d} y^{\text {jet }}$ for $\gamma+$ jet production is obtained from the number of data events in each interval after applying corrections for signal purity, acceptance and efficiency, divided by the integrated luminosity and the widths of the interval in the photon transverse momentum, photon rapidity ( $\left.\mathrm{d} y^{\gamma}=2.0\right)$, and jet rapidity ( $\left.\mathrm{d} y^{\mathrm{jet}}=1.6\right)$.

Figure 2 shows a comparison of the measured differential cross sections to the theoretical predictions from JETPHOX, PYTHIA, and SHERPA. There is a common $6.8 \%(11.2 \%)$ normalization uncertainty for central (forward) photons, not shown on the data points. The NLO predictions obtained using JETPHOX with CT10 PDF set [9] and BFG fragmentation functions of partons to photons [10]. The renormalization, factorization, and fragmentation scales $\mu_{R}=\mu_{F}=\mu_{f}$ are set equal to $p_{T}^{\gamma}$. The CT10 PDF uncertainties are estimated using 26 pairs of eigenvectors following the prescription of Ref. [11]. The JETPHOX predictions at the particle level are corrected for nonperturbative effects caused by parton-to-hadron fragmentation and MPI. Ratios of the JETPHOX predictions with MSTW2008NLO [12] and NNPDFv2.1 [13] PDF sets to those with CT10 PDF set are also presented.

The data agree with the $\mathrm{pQCD}$ NLO predictions with central photons in almost all jet rapidity regions except low $p_{T}^{\gamma}(<40 \mathrm{GeV})$ and the opposite-sign rapidity events at high $p_{T}^{\gamma}$ with very forward jets $\left(2.4<\left|y^{\mathrm{jet}}\right| \leq 3.2\right)$. They also describe data with forward photons except for the same-sign rapidity events with $p_{T}^{\gamma}>70 \mathrm{GeV}$ and $2.4<\left|y^{\mathrm{jet}}\right| \leq 3.2$. The measured cross sections typically have similar or smaller uncertainties than the NLO PDF and scale uncertainties. These measurements provide valuable information for tuning QCD theory predictions and particularly can be used as valuable input to global fits to gluon and other PDFs. 

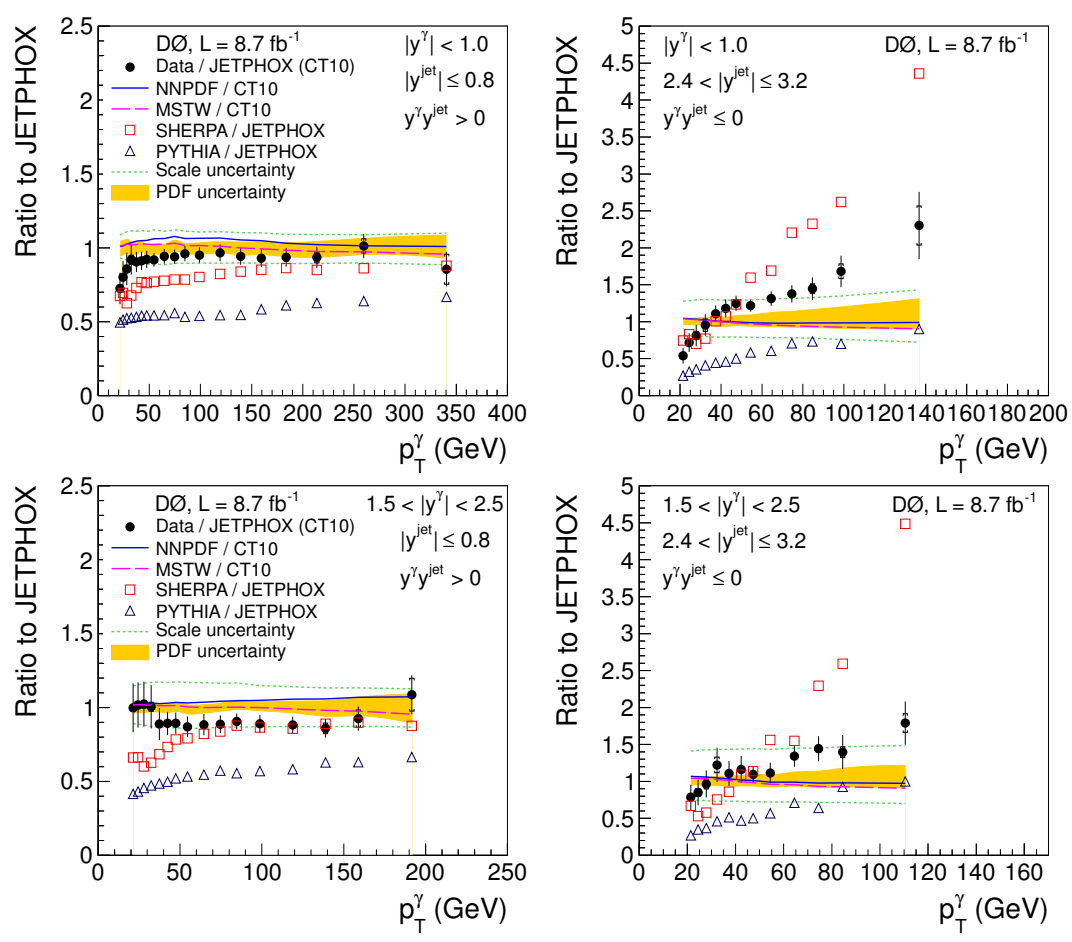

Figure 2: Ratios of the measured differential cross sections shown for central and forward photons, very central and very forward jet rapidities, same-sign and opposite-sign rapidity intervals to the pQCD NLO prediction using JETPHOX with the CT10 PDF set and $\mu_{R}=\mu_{F}=\mu_{f}=p_{T}^{\gamma}$. The solid vertical line on the points shows the statistical and pT-dependent systematic uncertainties added in quadrature, while the internal line shows the statistical uncertainty. The two dotted lines represent the effect of varying the theoretical scales of JETPHOX by a factor of two. The shaded region is the CT10 PDF uncertainty. The dashed and dashdotted lines show ratios of the JETPHOX predictions with MSTW2008NLO and NNPDFv2.1 to CT10 PDF sets. The predictions from SHERPA and PYTHIA are shown by the open squares and triangles, respectively.

\section{References}

[1] J. F. Owens, Rev. Mod. Phys. 59, 465 (1987).

[2] V. M. Abazov et al. (D0 Collaboration), Phys. Rev. D 88, 072008 (2013).

[3] S. Catani et al., J. High Energy Phys. 05, 028 (2002).

[4] T. Sjöstrand, S. Mrenna, and P. Z. Skands, J. High Energy Phys. 05, 026 (2006). We use PYTHIA version v6.420 with Tune A.

[5] T. Gleisberg et al., J. High Energy Phys. 02, 007 (2009). We use SHERPA version v1.3.1.

[6] V. M. Abazov et al. (D0 Collaboration), Nucl. Instrum. Methods Phys. Res. A 565, 463 (2006).

[7] G. C. Blazey et al., arXiv:hep-ex/0005012 (2000).

[8] R. J. Barlow and C. Beeston, Comp. Phys. Comm. 77, 219 (1993).

[9] H. L. Lai, et al., Phys.Rev. D 82, 074024 (2010).

[10] L. Bourhis, M. Fontannaz, and J. P. Guillet, Eur. Phys. J. C 2, 529 (1998).

[11] P. Nadolsky and Z. Sullivan, eConf C010630 (2001) P510, hep-ph/0110378.

[12] A. D. Martin et al., Eur. Phys. J. C 63, 189 (2009).

[13] R. D. Ball et al., Nucl. Phys. B 849, 296 (2011). 